Original Article

\title{
The effect of a pelvis-concentrated exercise program on male college students' body alignment and foot base pressure
}

\author{
Tae-Ho Kim, PT, PhD ${ }^{1)}$, Chae-Woo Lee, PT, PhD ${ }^{1)}$, Seong-Gil Kim, PT, PhD ${ }^{1)}$, \\ Byung-WoOK An, PT, MS ${ }^{1 *}$ \\ 1) Department of Physical Therapy, College of Rehabilitation Science, Daegu University: 15 Jillyang, \\ Gyeongsan-si, Gyeongbuk 712-714, Republic of Korea
}

\begin{abstract}
Purpose] The aim of this study was to examine the effect of a pelvis-concentrated exercise program and walking on the changes in body shape and foot base pressure. [Subjects and Methods] Thirty adults from $\mathrm{K}$ University in Busan, Republic of Korea, were randomly divided into the Swiss-ball exercise group and McKenzie exercise group, and they conducted exercise for $40 \mathrm{~min} 3$ times a week for 6 weeks. [Results] Global postural system results and foot base pressure significantly decreased in both groups. A comparison of foot base pressure after the intervention between the two groups revealed that the Swiss-ball exercise group exhibited a greater reduction than the McKenzie exercise group. [Conclusion] The results of this study indicated that the Swiss-ball exercise may improve posture and foot base pressure in male adults.

Key words: Pelvic exercise, Body alignment, Foot pressure
\end{abstract}

(This article was submitted Oct. 27, 2014, and was accepted Dec. 11, 2014)

\section{INTRODUCTION}

Since humans walk in an upright position, the spine is pressured by gravity and weight bearing. Therefore, the spine is faced with risky situations at all times, and lumbar herniated intervertebral disc occurs easily. This in turn causes weakened muscle strength, imbalanced muscle strength, and unstable joints and chronically worsens, affecting the whole spine $^{1,2)}$.

From the posture-balance point of view, the pelvis supports the abdomen and connects the spine to the lower limbs. When standing, the pelvis is an important part, as it supports weight from the spine to the lower limbs, allowing the trunk to be a stable foundation, and allows smooth movement of the upper limbs through maintenance of an upright posture. We can maintain an upright posture when the pelvis is located in a neutral position. Maintaining an upright posture also allows us to improve everyday movements and walking ability by adjusting the upper and lower limbs in dynamic postures $^{3)}$.

The lower limbs are connected to the acetabulums in the pelvis. Therefore, the pelvis affects lower limb movements. The location of the pelvis is also related to gait. When a person takes a step, the pelvis generates a biomechanical

*Corresponding author. Byung-Wook An (E-mail: ckck1@ hanmail.net)

C2015 The Society of Physical Therapy Science. Published by IPEC Inc. This is an open-access article distributed under the terms of the Creative Commons Attribution Non-Commercial No Derivatives (by-ncnd) License $<$ http://creativecommons.org/licenses/by-nc-nd/3.0/>. reaction as weight is loaded onto the lower limbs and saves energy consumed by gait ${ }^{4}$.

Most pelvis exercises would have different intensity compared with the therapeutic exercises prescribed for low back pain patients based on symptoms and pain. However, most exercises prescribed are a mixture of McKenzie exercises. Recently, more people are interested in Swiss-ball exercise, which is related to improvement of posture control ability and suitability of muscle activation. Swiss-ball exercise is easily accessible by all people, has wide range of applications using a Swiss ball, and has a high level of utility as a home exercise program. Exercise with a Swiss ball is used as a method of improving dynamic stabilization. It can improve muscle strength, endurance, and flexibility. Instability induced by a Swiss ball is reported to strengthen reflexes, perception ability, sense of balance, and proprioception comprehensively ${ }^{5,6)}$

In particular, the shift of the center of pressure (COP) is utilized as an evaluation tool for diagnosis and treatment of balance disability ${ }^{7}$. Force platform, pressure plate, and in-shoe pressure measurement can be used to measure foot pressure. They can detect pressures from various points on the foot in various forms and provide timely and quantitative data.

In this we study, we assumed that an abnormal posture habit of a normal adult can cause problems in trunk muscles and affect posture alignment, foot base pressure, and balancing ability. Therefore, we examined the effect of a pelvisconcentrated exercise program and walking on the changes in body shape and foot base pressure. 
1166 J. Phys. Ther. Sci. Vol. 27, No. 4, 2015

Table 1. General characteristics of the subjects

\begin{tabular}{lrr}
\hline & \multicolumn{1}{c}{ SEG } & \multicolumn{1}{c}{ MEG } \\
\hline Age (years) & $23.9 \pm 2.2$ & $21.1 \pm 1.9$ \\
Height $(\mathrm{cm})$ & $172.6 \pm 5.7$ & $161.56 \pm 4.7$ \\
Weight $(\mathrm{kg})$ & $69.1 \pm 10.8$ & $54.9 \pm 9.7$ \\
\hline
\end{tabular}

Mean \pm SD. SEG: Swiss-ball exercise group; MEG:

McKenzie exercise group

\section{SUBJECTS AND METHODS}

This study utilized 30 male and female students in their 20s and 30s from K University, Busan, Republic of Korea. The subjects were divided into a Swiss-ball exercise group and a McKenzie exercise group. Each group exercised for 40 minutes a day 3 times a week for 6 weeks. The subjects satisfied the following conditions: 1) they did not exercise, 2 ) they had not had any accidents or continuous damages on lower limbs in the last 6 months, 3) they had no history of neurological diseases, decreased coordination capability, or damage to motor sensation, 4) they had no history of musculoskeletal diseases, and 5) they did not have any lesions or skin diseases on their feet. All the subjects understood the purpose of this study and provided written informed consent prior to participation in the study in accordance with the ethical standards of the Declaration of Helsinki (Table 1).

The Swiss-ball exercise group sat on a Swiss ball and performed the following movements: 1) they put their two hands on the back of their head, touched the ball with their back, forming a bridge position, and pulled and pushed the ball with their feet. 2) They put their arms on their chest, fixed the trunk as steady as possible, and drew a circle by spinning their pelvis. 3) They put their calves on the ball in a lying position and repeatedly bent and stretched the knee joints and hip joints.

The McKenzie exercise group performed the following movements: 1) They put one side of their head on the floor, put their arms at their sides, and took a deep breath 3 to 4 times. 2) In a prone position, they pulled their knees without applying too much pressure. 3) Again in a prone position, they extended their upper body with their weight on their elbows and forearms. 4) Again in a prone position, they extended their upper body with their weight on their arms and their arms straight. 5) In a standing position, they leaned backwards as far as possible. 6) They sat on a chair, bent at their waist, put their palms on the ground, and then returned to the original position.

This study used a global postural system (GPS, LAXTHA) to measure changes in the anatomic alignments related to the baseline set for pelvis tilting and the change in the distribution of foot base pressure (Alfoots, Seoul, Republic of Korea). All the results of measurement are expressed as the mean \pm standard deviation. PASW Statistics for Windows (version 18.0) was used for data analyses. The paired t-test was used to analyze the difference between before and after the intervention, and the independent t-test was used to see the differences in results of each group. The statistical significance level was $\alpha=0.05$.
Table 2. Comparison of pre- and post-intervention measurement values

\begin{tabular}{lccc}
\hline \multicolumn{1}{c}{ Variable } & Group & Pre & Post \\
\hline GPS & SEG & $1.6 \pm 0.4$ & $1.2 \pm 0.2^{\mathrm{a}}$ \\
$\left(^{\circ}\right)$ & MEG & $1.7 \pm 0.3$ & $1.4 \pm 0.2^{\mathrm{a}}$ \\
Static foot & SEG & $3.2 \pm 2.1$ & $1.4 \pm 1.1^{\mathrm{a}}$ \\
pressure (\%) & MEG & $4.1 \pm 2.4$ & $2.1 \pm 1.5^{\mathrm{a}}$ \\
Dynamic foot & SEG & $4.1 \pm 3.8$ & $1.6 \pm 1.0^{\mathrm{ab}}$ \\
pressure $(\%)$ & MEG & $3.9 \pm 2.2$ & $2.1 \pm 1.4^{\mathrm{ab}}$ \\
\hline
\end{tabular}

aSignificant difference between pre- and post-intervention values. bSignificant difference in post-intervention values between SEG and MEG

\section{RESULTS}

Both the groups showed a significant decrease in the GPS results after the intervention $(\mathrm{p}<0.05)$ and in foot base pressure $(\mathrm{p}<0.05)$. When the two groups were compared after the intervention, the Swiss-ball exercise group showed a bigger decrease in foot base pressure compared with the McKenzie exercise group $(\mathrm{p}<0.05)$ (Table 2$)$.

\section{DISCUSSION}

Weakened and imbalanced deep muscles cause decreased reposition sense ability due to lowered proprioceptive function. This is reported to be a cause of recurrence of low back pain, as problems occur in spinal stability ${ }^{8}$. Overload of passive systems such as the lumbar herniated intervertebral disc especially increases the rate of active system load as a compensation for stress. In this case, strengthening of the active system is very important for maintaining spinal stability $^{9)}$.

Many clinical studies suggest that there are problems with pelvic floor muscle exercise therapy. Since the pelvic floor muscle is not a muscle that we use every day, it is reported that pelvic floor muscle contraction exercise cannot be performed properly by some patients only based on a therapist's explanation. Therefore, it is important to develop various pelvic floor muscle exercise programs with accurate contraction methods based on a thorough understanding of the role of pelvic floor muscles for effective waist stabilization $^{10)}$.

The swiss ball is a very good exercise tool for therapeutic rehabilitation exercise and reeducating exercise nerves in neurological way. Swiss-ball exercise is an interesting exercise, and anyone can perform the necessary exercises safely. It can develop flexibility and stability of the spine, as it is possible to train muscles, joints, and sense of equilibrium with tens of postures, including sitting and lying downward on the ball.

In this study, the subject groups performed pelvis-concentrated exercise. Comparison of the pre- and post-exercise measurement results in each group showed that foot base pressure in both groups decreased to close to the normal level. In this study, the Swiss-ball exercise group had a slightly higher correction rate compared with the other group. This is because the Swiss-ball exercise has detailed goals such 
as improving muscle strength, endurance, coordination, movability, and flexibility in addition to maintenance and improvement of joint range of motion.

As the pelvis-concentrated exercise was included in intensive training, the contacting and bearing surfaces were increased. Therefore, movement of trunk and pelvis increased, which in turn reduced the distribution of the foot base pressure in the standing position.

As the distribution of the foot base pressure is stabilized by a stable trunk, body balance improves, which in turn distributes the right-side and left-side weight more effectively.

It has been reported that pressure on certain parts of the foot can be observed when performing various daily motions and functional activities ${ }^{11)}$, and this is one of the subjects that should be investigated in the field of sports science.

According to the results concerning posture alignment and foot base pressure distribution, both exercise programs had a positive effect on posture change and foot base pressure. Of the two programs, Swiss-ball exercise had a better effect. Therefore, a pelvis-concentrated exercise program is effective in maintaining a correct posture as well as in improving balance and exercise ability. It can also be utilized as an abnormal posture prevention program. More clinical application and continuous research are expected so that it can be standardized and studied more in depth.

\section{REFERENCES}

1) Armstrong JR: Lumbar Disc lesions: Pathogenesis and Treatment of Low Back Pain and Sciatica. Williams and Wilkins, 1965.

2) Lee CW, Hyun J, Kim SG: Influence of pilates mat and apparatus exercises on pain and balance of businesswomen with chronic low back pain. J Phys Ther Sci, 2014, 26: 475-477. [Medline] [CrossRef]

3) Kapandji I: The Physiology of theJoints, vol. III. The Trunk and the Vertebral Column. Edinburgh London and New York. Churchill Livingstone, 1974.

4) Norkin C, Levangie P: Joint Structure and Function. FA. Davis, 1992.

5) Janda V: The Swiss Ball Theory, Basic Exercises and Clinical Application. USA: Springer, 1998.

6) Mori A: Electromyographic activity of selected trunk muscles during stabilization exercises using a gym ball. Electromyogr Clin Neurophysiol, 2004, 44: 57-64. [Medline]

7) Rocchi L, Chiari L, Cappello A, et al.: Comparison between subthalamic nucleus and globus pallidus internus stimulation for postural performance in Parkinson's disease. Gait Posture, 2004, 19: 172-183. [Medline] [CrossRef]

8) O'Sullivan PB, Burnett A, Floyd AN, et al.: Lumbar repositioning deficit in a specific low back pain population. Spine, 2003, 28: 1074-1079. [Medline] [CrossRef]

9) Norris C: Functional load abdominal training: part 1. J Bodyw Mov Ther, 1999, 3: 150-158. [CrossRef]

10) Harvey MA: Pelvic floor exercises during and after pregnancy: a systematic review of their role in preventing pelvic floor dysfunction. J Obstet Gynaecol Can, 2003, 25: 487-498. [Medline]

11) Kernozek T, LaMott E: Comparisons of plantar pressures between the elderly and young adults. Gait Posture, 1995, 3: 143-148. [CrossRef] 